\title{
Variation in oxytocin is related to variation in affiliative behavior in monogamous, pairbonded tamarins
}

\author{
Charles T. Snowdon *, Bridget A. Pieper, Carla Y. Boe, Katherine A. Cronin, Aimee V. Kurian, Toni E. Ziegler \\ Department of Psychology, University of Wisconsin, Madison, Wisconsin National Research Center, Madison WI, USA
}

\section{A R T I C L E I N F O}

\section{Article history:}

Received 29 March 2010

Revised 18 June 2010

Accepted 23 June 2010

Available online 28 June 2010

\section{Keywords}

Oxytocin

Affiliative behavior

Cotton-top tamarins

Monogamy

Cooperative breeding

Sex differences

\begin{abstract}
A B S T R A C T
Oxytocin plays an important role in monogamous pairbonded female voles, but not in polygamous voles. Here we examined a socially monogamous cooperatively breeding primate where both sexes share in parental care and territory defense for within species variation in behavior and female and male oxytocin levels in 14 pairs of cotton-top tamarins (Saguinus oedipus). In order to obtain a stable chronic assessment of hormones and behavior, we observed behavior and collected urinary hormonal samples across the tamarins' 3-week ovulatory cycle. We found similar levels of urinary oxytocin in both sexes. However, basal urinary oxytocin levels varied 10 -fold across pairs and pair-mates displayed similar oxytocin levels. Affiliative behavior (contact, grooming, sex) also varied greatly across the sample and explained more than half the variance in pair oxytocin levels. The variables accounting for variation in oxytocin levels differed by sex. Mutual contact and grooming explained most of the variance in female oxytocin levels, whereas sexual behavior explained most of the variance in male oxytocin levels. The initiation of contact by males and solicitation of sex by females were related to increased levels of oxytocin in both. This study demonstrates within-species variation in oxytocin that is directly related to levels of affiliative and sexual behavior. However, different behavioral mechanisms influence oxytocin levels in males and females and a strong pair relationship (as indexed by high levels of oxytocin) may require the activation of appropriate mechanisms for both sexes.
\end{abstract}

(c) 2010 Elsevier Inc. All rights reserved.

\section{Introduction}

Oxytocin is a peptide hormone that has been shown to be involved in pair-bonding and social memory (reviewed by Carter, 1998). There are clear species differences with distribution of oxytocin receptors and differential effects of oxytocin agonists and antagonists between monogamous and polygamous species of voles. There are also clear sex differences with neural mechanisms of pair-bonding in monogamous female rodents being influenced by differential sensitivity to exogenous oxytocin and/or differential oxytocin responsiveness to specific stimuli whereas males appear similarly influenced by responsiveness to the closely related peptide, arginine vasopressin (e.g. Carter, 1998; Insel and Shapiro, 1992; Lim and Young, 2006). However, detailed studies of the distribution of oxytocin immunoreactive nerve cells and fibers in the brains in both socially monogamous prairie voles and common marmoset (Callithrix jacchus) found no sex differences (Wang et al., 1996, 1997). On the other hand measures of peripheral oxytocin in

\footnotetext{
* Corresponding author. Department of Psychology, University of Wisconsin, 1202 West Johnson Street, Madison, WI 53706, USA. Fax: + 16082624029.

E-mail address: Snowdon@wisc.edu (C.T. Snowdon).
}

both polygynous and monogamous species of rodents reported higher concentrations in females (Kramer et al., 2004).

Several studies have indicated that oxytocin levels are affected by sexual and affiliative behavior. Newly paired prairie voles and cottontop tamarins engage in extensive sexual activity (Carter, 1998; Savage et al., 1988) and in both men and women peripheral oxytocin levels are elevated with orgasm (Krüger et al., 2003). Tactile stimulation such as massage and hugging also leads to elevated oxytocin levels in both humans and rodents (Uvnäs-Moberg, 1998; Light et al., 2005), and intranasal administration of OT has been shown to have several effects in humans including increased trust and attachment behavior (e.g. Kosfeld et al., 2005; Zak et al., 2005). In humans, Grewen et al. (2005) reported elevations of oxytocin in both men and women with strong partner support. However, measures of relationship distress correlated with oxytocin levels in women and with arginine vasopressin levels in men (Taylor et al., 2010).

These results suggest that natural variation in touch and sexual activity along with relationship quality might be associated with individual variation in oxytocin levels. Few studies have looked at the effects of within-species variation in affiliative and sexual behavior on oxytocin levels in both sexes. The goals of the current study were to examine possible sources of within-species variation in oxytocin levels by simultaneously measuring affiliative and sexual behavior and oxytocin levels over the course of a complete ovarian cycle. We used a 
complete ovarian cycle in order to establish chronic or basal measures of both hormones and behavior rather than note acute responses to specific events. We studied cotton-top tamarins (Saguinus oedipus), a cooperatively breeding species closely related to the common marmoset. Based on the fact that we have found few behavioral sex differences in tamarins, we predicted that there would be no differences between sexes in levels of oxytocin. We further predicted that oxytocin levels within a pair would be highly correlated. We also predicted that some of the variance in oxytocin levels would be explained by variation in affiliative and sexual behavior.

\section{Methods}

\section{Subjects and housing}

The subjects were 14 male-female pairs of cotton-top tamarins that had lived together for more than a year. One animal in each pair was sterilized for colony management reasons, but all had intact gonads. No offspring were present for any pair. Pairs were housed in identical cages constructed of anodized aluminum and poly-urethane coated mesh measuring $160 \times 236 \times 90 \mathrm{~cm}(L \times H \times W)$. Cages were fitted with branches and ropes to simulate the natural arboreal environment. The animals received three feedings each day: a snack as they awoke in the morning, a main feed at midday and a high protein snack in late afternoon. For additional details of husbandry see Ginther et al. (2001).

\section{Data collection}

\section{Hormonal}

Urine samples were collected three times each week over a 3-week period to encompass the complete ovarian cycle of the female (Ziegler et al., 1987). Animals were awakened by turning on the lights and a person entered the cage and held a polypropylene container under the target monkeys to obtain the first morning void urine sample. Urine samples from each member of a pair were collected on the same mornings. All animals were well habituated to this procedure. As soon as samples were collected they were centrifuged and then aliquoted into two samples, one for steroid analyses and the other for peptide analyses. Samples were immediately stored at $-20{ }^{\circ} \mathrm{C}$ until the time of assay.

\section{Behavioral}

We completed a 30-min behavioral observation on each pair three times a week. Data were collected on a laptop computer using Observer 5.0 software (Noldus). We classified behavior into two broad categories, mutual behaviors that involved both members of the pair (and were thus scored for the pair rather than the individual, except grooming, see below), and individual behaviors. Mutual affiliative behaviors were huddling (animals in body contact with tails curled, duration and frequency recorded), proximity (within a tamarin arm's reach, duration recorded) and grooming (identity of groomer, duration, and frequency of bouts recorded). Mutual sexual behaviors included solicitations by both sexes and all mounts by males whether successful or not. Individual behaviors included female solicitations (vertical stretch directed toward mate with body rubbing on substrate), erections (by males), headshaking and tongue flicking, investigation (sniffing or licking of genitals of mate), initiation of proximity, grooming by one individual, female scent marks, male investigation of scent marks (within $5 \mathrm{~s}$ of female marking). Observation sessions alternated between morning and afternoon with nearly equal numbers at each time and were carried out prior to the main feed at noon or at least 1 hour following the main feed and before the afternoon snack. All observers were trained to a criterion of $>85 \%$ reliability prior to observations.

\section{Hormonal assays}

\section{Oxytocin}

There is evidence suggesting that some neuropeptides can cross the blood-brain barrier in both neonatal (e.g. Bales and Carter, 2003) and adult rodents (e.g. Banks and Kastin, 1985) and that peripheral measures of oxytocin can correlate with the biobehavioral functions of oxytocin (Kramer et al., 2004; Ring et al., 2006). Functional changes in urinary oxytocin have been measured in human children in response to tactile stimulation from mothers (Wismer Fries et al., 2005), in adult human males while interacting with infants (Gray et al., 2007) and in common marmosets with differences between socially isolated and non-isolated animals (Seltzer and Ziegler, 2007). The assays used in the current study were modified slightly from the previously used radioimmunoassay (RIA). A direct enzyme immunoassay (EIA) (Assay Designs, Ann Arbor, MI) has been shown to have greater specificity than the earlier RIA, responding only to the fraction eluded at the location of oxytocin (Carter et al., 2007). EIA reactivity with arginine vasopressin was less than $0.3 \%$.

Samples were run in duplicate at a volume of $200 \mu$ l. Urine was diluted to $1 \mathrm{ml}$ with purified water and $10 \mu \mathrm{l}$ of phosphoric acid and purified by solid phase extraction (SPE). The SPE columns (Phenomenix Strata X) were conditioned with $1 \mathrm{ml}$ methanol and $1 \mathrm{ml}$ purified water. The samples were added and washed with $10 \%$ acetonitrile, $1 \%$ TFA (trifluoroacetic acid) in water and eluted with $1 \mathrm{ml} \mathrm{80:20}$ acetonitrile: water. Samples were dried and resuspended in $200 \mu \mathrm{l}$ assay buffer and added to the microtiter plates according to the directions provided with the assay kit. All concentrations were divided by creatinine levels to control for variation in urine concentration. Values are expressed in $\mathrm{pg} / \mathrm{mgCr}$. Serial diluted pooled tamarin urine was parallel to oxytocin standards $(t=1.21, P>0.05)$ and accuracy was $101.9+4.7 \%($ mean + SEM $)$. The upper sensitivity limit of the oxytocin assay was $500 \mathrm{pg} / \mathrm{ml}$ and values registering higher than this were entered as $500 \mathrm{pg} / \mathrm{ml}$. Mean intra-assay coefficients of variation were $5.85 \%$ and inter-assay coefficients of variation were $16.11 \%$.

\section{Biological validation}

We completed two biological validations in tamarins of the oxytocin assays. We implanted three males with an estradiol pellet $(1.5 \mathrm{mg}$ continuous release over 30 days) and we collected urine samples before and during the period of the implant. Estradiol increased significantly in all three males $(t=3.64, P=0.04)$ ensuring the effectiveness of the pellets in raising estradiol. Male oxytocin response varied substantially, but two of the three males showed a large increase in oxytocin following the estradiol elevation (mean pre $\mathrm{OT}=17 \pm 5 \mathrm{pg} / \mathrm{mg} \mathrm{Cr}$, mean post $\mathrm{OT}=100 \pm 15 \mathrm{pg} / \mathrm{mg} \mathrm{Cr}$ ) (Fig. 1). These data are consistent with what is known about the stimulatory effect of estradiol on oxytocin (Ochedalski et al., 2007; Pedersen and Prange, 1979) and indicate that functional changes in oxytocin can be measured in urine.

We also compared urinary oxytocin levels in four tamarins housed alone for 1 week and with the same animals during the first week after pairing with a mate. We found a significant increase in oxytocin after pairing $(23 \pm 26.3 \mathrm{pg} / \mathrm{mg} \mathrm{Cr}$ alone, $100 \pm 2.7 \mathrm{pg} / \mathrm{mg} \mathrm{Cr}$ after pairing, $p<0.003$ ), as would be expected based on known patterns of oxytocin release. We also collected a urine sample from one male immediately after mounting and observed a level of oxytocin similar to the levels seen in the week following pairing. Thus the urinary oxytocin assay provides results that would be expected following physiologically and behaviorally induced oxytocin release and results are similar to patterns following manipulation of social conditions by Seltzer and Ziegler (2007) in the related common marmoset.

\section{Cortisol}

We measured cortisol levels according to the methods of Ziegler et al. (1995), but found no significant relationships between cortisol and oxytocin or cortisol and behavior and do not discuss this further. 

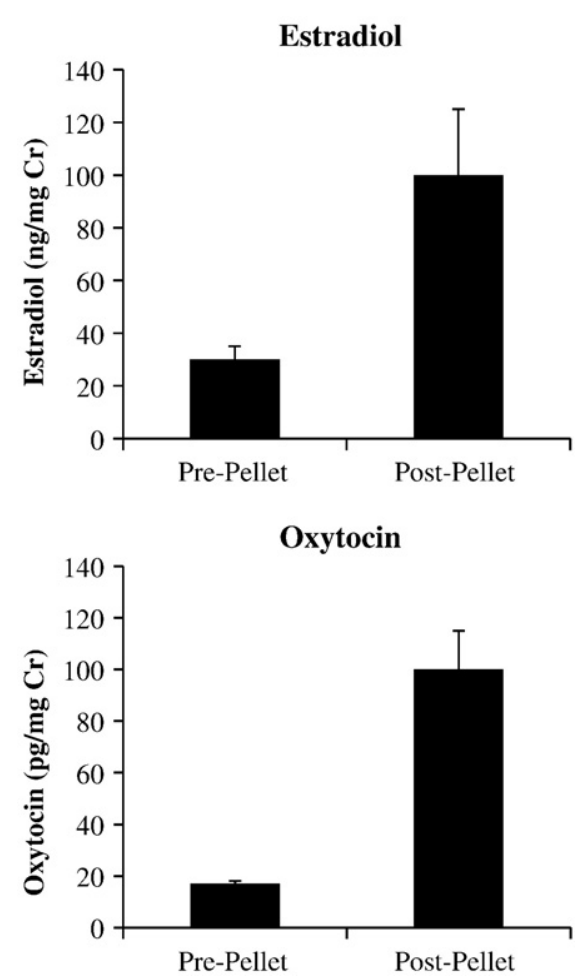

Fig. 1. Hormonal response of male tamarins to estrogen pellets. Top: estradiol, Bottom: oxytocin.

\section{Data analyses}

Our goal was to provide an assessment of chronic (or basal) levels of both oxytocin and behavior rather than to detect acute responses to individual events. Hence we used assays of first morning urinary samples that integrated hormonal activity over the preceding $13 \mathrm{~h}$ or more and we averaged hormonal and behavioral measures across the 3-week ovarian cycle of the female (Ziegler et al., 1987). Behavioral data included both frequency and duration measures. In order to obtain a global comparison of oxytocin levels with affiliative behaviors, we first rank ordered pairs separately for duration and frequency of huddling and duration and frequency of grooming and frequency of sexual behaviors. We then averaged these ranks to obtain one rank value per pair. We also calculated pair ranks excluding sexual behavior to measure affiliation independently. We used Spearman rank order correlations to compare oxytocin levels of males and females within a pair and to compare the mean rank of each pair with levels of affiliative and sexual behavior. We used a $t$-test to determine if there were any differences in oxytocin levels between males and females. Finally, we used the raw data from paired measures (huddling and grooming duration, huddling and grooming frequency, and frequency of sexual behavior) and individual measures in stepwise multiple regression analyses (SPSS 16.0) to determine which components influenced variance in mean pair oxytocin and variance in female and male oxytocin levels taken separately.

\section{Results}

Male and female oxytocin levels did not differ across the sample (mean \pm SEM: female $23.69 \pm 5.25$, male $16.26 \pm 3.56 \mathrm{pg} / \mathrm{mg}$ $\mathrm{Cr}, t(26)=1.17, P=0.25)$. There was marked variation between individuals within each sex in mean basal oxytocin levels with a 10-fold range of values for both females (4.5-46.4 pg/mg Cr) and males (3.89$39.92 \mathrm{ng} / \mathrm{mg} \mathrm{Cr}$ ). However, within pairs there was a close correspondence between male and female oxytocin levels with a paired-sample $t$ test showing no significant sex difference $(t(13)=1.35, P=0.199)$ and a significant Spearman rank order correlation between mates $\left(R_{\mathrm{S}}=0.714\right.$, $n=14, P=0.02$ ) (Fig. 2). The striking within-species variation also appeared for the behavioral measures with sexual behavior ranging from 1.5 to 6.9 acts per $30 \mathrm{~min}$, huddling and grooming duration ranging from 14.6 to 556.8 s per $30 \mathrm{~min}$ and huddling and grooming frequency ranging from 1.9 to 18.2 acts per 30 min.

We compared pair mean ranks of sexual behavior and huddling and grooming frequency and duration to the rank order of mean pair oxytocin levels. Pair oxytocin levels correlated significantly with affiliative behavior $\left(R_{\mathrm{S}}=0.783, n=14, P<0.01\right.$; Fig. 3$)$. When sexual behavior was removed as a variable using only the rank order based on huddling and grooming frequency and duration the correlation with pair oxytocin rank was still highly significant $\left(R_{\mathrm{S}}=0.72, n=14\right.$, $P<0.02$ ).

To further identify which behaviors were best predictors of within-pair variation and male and female variation in oxytocin separately we completed stepwise multiple regression analyses. The model that explained the greatest variance in mean pair oxytocin levels included both total sexual behavior and frequency of affiliative behavior $(F(2,11)=9.54, P=0.004$, adjusted $r$ square $=0.57$ beta for sexual behavior $=0.50, t=2.55, P=0.03$, for frequency of affiliation behavior, beta $=0.47, t=2.397, P=0.04)$. For predictors of variance in female oxytocin levels the model excluded sex but included both affiliation duration and affiliation frequency $(F(3,10)=3.937$, $P=0.04$ adjusted $r$ square $=0.40$ ). The beta coefficient for affiliation frequency was $0.62(t=2.325, P=0.04)$; for affiliation duration beta $=0.56(t=2.188, P=0.054)$. When predictors of male oxytocin levels were tested, the significant model included sexual behavior as the only significant variable $(F(1,12)=7.46, P=0.02$, adjusted $r$ square $=0.33$; beta coefficient for sexual behavior $=0.62, t=2.731$, $P=0.02)$.

When we evaluated behaviors exhibited by individuals as contributors to variance in pair oxytocin levels three models emerged. The model that explained the most variance included male sexual behavior, male huddle initiation and female solicitation $(F(3,10)=21.54$, $P=.001$, adjusted $r$ square $=0.83$, beta for sexual behavior $=0.38$, $t=2.80 P=.02$, beta for male huddle initiate $=0.62, t=4.85, P=0.001$, and beta for female solicit $=0.45, t=3.43, P=0.006$.).

The role of individual behavior in explaining variation in female oxytocin yielded two models. The model that explained the greatest variance included male huddle initiation and female solicitation $(F(2,11)=8.53, P=0.006$, adjusted $r$ square $=0.54$, beta for male huddle initiate $=0.73, t=3.783, P=0.003$, beta for female solicit $=0.45$, $t=2.34, P=0.04$ ). One significant model (male erections) explained variance in male oxytocin levels $(F(1,12)=10.69, P=0.006$, adjusted $r$ square $=0.43$, beta for erection $=0.69, t=3.31, P=0.006$ ).

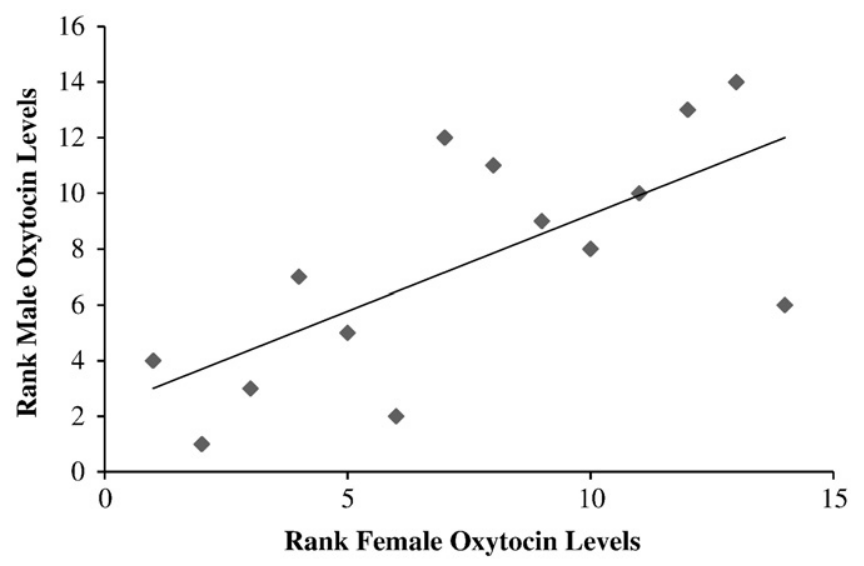

Fig. 2. Within pair correlation of oxytocin levels. $R_{\mathrm{S}}=0.71, p<0.02$. 


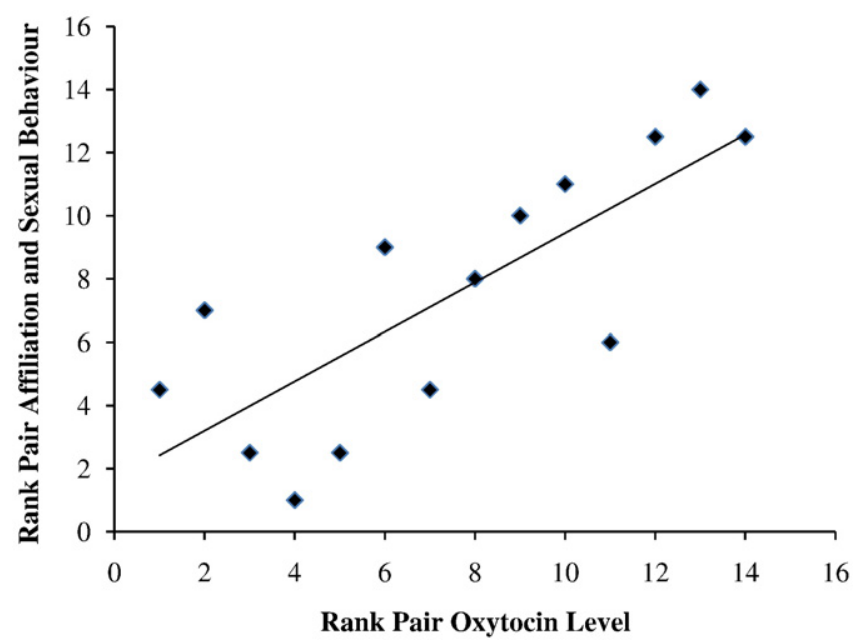

Fig. 3. Correlation of pair oxytocin levels with affiliative and sexual behavior. $R_{\mathrm{S}}=0.78$, $p<0.01$.

\section{Discussion}

We examined the levels of urinary oxytocin of 14 pairs of cottontop tamarins over the 3-week ovulatory cycle of females, and we simultaneously sampled affiliative and sexual behavior within each of the pairs. In contrast to previous research on rodents (polygynous rats and monogamous prairie voles) where sexually naïve females had increased levels of oxytocin compared to sexually naïve males (Kramer et al., 2004) we found no sex differences in oxytocin levels. Wang et al. (1997) studied the distribution of oxytocin immunoreactive neurons and fibers in common marmosets, a closely related species, and found no sex differences in the distribution of oxytocin immunoreactive cells. In both marmosets and tamarins sexual roles are not highly differentiated, with both taking part in infant care, vigilance and territory defense, which may be related to the lack of sex differences in peripheral oxytocin levels. However, our data suggest that oxytocin levels are stimulated by different behavioral variables for each sex.

An unexpected result was the 10-fold variation in oxytocin levels across individuals. The levels and brain distribution of oxytocin are known to differ significantly between monogamous versus promiscuous breeding species of voles but we are aware of only one study demonstrating such high levels of within species variation (Levine et al., 2007, in humans). Hammock and Young $(2002,2005)$ reported within species differences in AVP1A receptor genes and social behavior in prairie vole males, and a recent study on humans found similar variation in AVP1A receptor genes and male pair-bonding behavior (Walum et al., 2008). We also found corresponding variation in measures of affiliative behavior across pairs. Our results suggest that variation in affiliative and sexual behavior may have a significant effect on hormones or vice versa. This variation in hormones and behavior within species has clear implications for understanding human behavioral variation in affiliation and pair bonding.

We also found a significant correlation between oxytocin levels in both males and females within tamarin pairs that suggests that complementary behavioral interactions may be responsible for the shared oxytocin levels. Some studies have shown that sexual activities as well as gentle stroking and touching can elicit increased oxytocin levels in humans (Uvnäs-Moberg, 1998; Krüger et al., 2003; Light et al., 2005). Our multiple regression analyses identified two pair behavioral components (sexual behavior and frequency of affiliative contact) that explained $60 \%$ of the variance in the mean oxytocin levels within pairs. In addition three individual behaviors, male sex, male initiation of huddling and female solicitation, explained nearly $83 \%$ of the variance in pair oxytocin levels These results suggest that these behaviors may have a tonic effect on oxytocin levels, and that peripheral oxytocin levels reflect the quality of a pair relationship. It is important to emphasize that the data presented here represent chronic (or baseline) levels of hormones and behavior. We do not know whether or how quickly oxytocin levels may change in response to sexual behavior or female fertility.

Other studies have shown that social interactions can influence oxytocin levels and vice versa. Grewen et al. (2005) reported higher plasma oxytocin levels in men and women with strong partner support. However, Ditzen et al. (2007) failed to show differences in oxytocin as a function of partner social support in response to a stress test. Gonzaga et al. (2006) reported that the number of affiliation (but not sexual) cues produced correlated with plasma oxytocin levels in women. Gordon et al. (2008) reported that oxytocin levels in romantically unattached young adults were positively correlated with bonding with their parents and negatively correlated to psychological distress. Oxytocin levels across pregnancy predicted post-partum mother-infant bonding (Feldman et al., 2007) and mothers with secure attachment style had greater plasma oxytocin response to infant cues (Strathearn et al., 2009). In contrast, Taylor et al. (2010) found that measures of relationship distress correlated with oxytocin levels in women but with arginine vasopressin levels in men. Studies of newly paired guinea pigs found correlations of plasma oxytocin levels within pairs with social grooming, and sexual behavior predicting female oxytocin levels on day 4 after pairing (Wallner et al., 2006). Odendaal and Meintjes (2003) have reported that affiliative interactions (petting and scratching) between humans and dogs increased oxytocin levels in both species. Our results suggest that chronic levels of oxytocin may be maintained through high levels of affiliative and sexual behavior.

Although we found no sex differences in oxytocin levels, multiple regression analyses showed that variation in oxytocin levels was best explained by different variables in each sex. For males, frequency of pair sexual behavior explained $45 \%$ of the variance and male erections explained $43 \%$ of the variance. For females the best model excluded sexual behavior but showed that $40 \%$ of the variance in female oxytocin could be explained by the frequency and duration of contact behavior and grooming. These results based on pair behaviors suggest males and females have complementary behavioral strategies with female oxytocin levels being influenced by amount of proximity and contact they receive whereas male levels are affected by amount of sexual activity. It is interesting that analyses of individual behaviors suggest that mates may be responsive to each other's needs. Male initiation of huddles with females and female solicitation of sex were both important variables in explaining variation in both pair and female oxytocin levels. A good relationship between pair-mates may be one where each sex can respond to the behavioral needs of its mate.

The natural establishment of pairbonds in prairie voles is usually accompanied by extensive sexual behavior over 1-2 days (Carter, 1998) which also induces ovulation in females. However, pairbonds can also be formed without extensive sexual behavior In tamarins sexual behavior may be important in pair formation as we have observed high rates of sexual behavior in newly formed pairs (Savage et al., 1988). However, tamarins also engage in high rates of nonconceptive sex not only over the female ovarian cycle but during pregnancy as well. We have reported (Snowdon and Ziegler, 2007) that rates of nonconceptive sex and female solicitation increase after minor threats to the relationship (brief separations, and olfactory stimuli from novel reproductive females) suggesting that nonconceptive sex may be important in restoring or maintaining a relationship. In support of this experimental studies on humans have demonstrated that oxytocin administered peripherally can increase perceptions of attractiveness and trust (Theodoridou et al., 2009), facilitate recognition of positive words associated with both sex and relationships (Unkelbach et al., 2008), enhance feelings of attachment security in 
men (Buchheim et al., 2009), and increase processing of positive emotional information in men (Di Simplicio et al., 2009).

The extensive within species co-variation in affiliation, sexual activity and oxytocin levels in tamarins suggests that among pairbonded primates there can be considerable within species variation. We suggest that the amount and quality of close contact and nonconceptive sex may also play an important role in predicting the quality and duration of human heterosexual relationships. The demonstration that both sexes of tamarins have equal levels of oxytocin within a pair, but that these levels are influenced by different behaviors for each sex and that each sex engages in behavior that complements the needs of the mate suggest ways in which human relationships might be improved.

\section{Acknowledgments}

Supported by USPHS Grant MH035215 and the University of Wisconsin Graduate School Research Committee and in part by support from the Wisconsin National Primate Research Center, RR000167, a component of the National Institutes of Health. Research protocol was approved by the University of Wisconsin, College of Letters and Science Animal Care and Use Committee. We thank two anonymous reviewers for valuable suggestions that have improved this manuscript.

\section{References}

Bales, K.L., Carter, S.C., 2003. Sex differences and developmental effects of oxytocin on aggression and social behavior in prairie voles (Microtus ochrogaster). Horm. Behav. $44,178-184$.

Banks, W.A., Kastin, A.J., 1985. Permeability of the blood-brain barrier to neuropeptides: the case for penetration. Psychoneuroendocrinology 10, 385-399.

Buchheim, A., Heinrichs, M., George, C., Pokorny, D., Koops, E., Henningesen, P., O'Connor, M.F., Gündel, H., 2009. Oxytocin enhances the experience of attachment security. Psychoneuroendocrinology 14, 1417-1422.

Carter, C.S., 1998. Neuroendocrine perspectives on social attachment and love. Psychoneuroendocrinology 23, 779-818.

Carter, C.S., Pournajafi-Nazarloo, H., Kramer, K.M., Ziegler, T.E., White-Traut, R., Bello, D., Schwertz, D., 2007. Oxytocin: behavioral associations and potential as a salivary biomarker. Ann. NY Acad. Sci. 1098, 312-322.

Di Simplicio, M., Massey-Chase, R., Cowan, P.J., Harmer, C.J., 2009. Oxytocin enhances processing of positive versus negative emotional information in healthy male volunteers. J. Psychopharm. 23, 241-248.

Ditzen, B., Neumann, I.D., Bodenmann, G., van Dawans, B., Turner, R.A., Ehlert, U., Heinrichs, M., 2007. Effects of different kinds of couple interaction on cortisol and heart rate responses to stress in women. Psychoneuroendocrinology 32, 565-574.

Feldman, R., Weller, A., Zagoory-Sharon, O., Levine, A., 2007. Evidence for a neuroendocrinological foundation of human affection: plasma oxytocin levels across pregnancy and the postpartum period predict mother-infant bonding. Psych. Sci. 18, 965-970.

Ginther, A.J., Ziegler, T.E., Snowdon, C.T., 2001. Reproductive biology of captive male cottontop tamarin monkeys as a function of social environment. Anim. Behav. 61, 65-78.

Gonzaga, G.C., Turner, R.A., Keltner, D., Campos, B., Altemus, M., 2006. Romantic love and sexual desire in close relationships. Emotion 6, 163-179.

Gordon, I., Zagoory-Sharon, O., Schniederman, I., Leckman, J.F., Weller, A., Feldman, R., 2008. Oxytocin and cortisol in romantically unattached young adults: associations with bonding and psychological distress. Psychophys. 45, 349-352.

Gray, P.B., Parkin, J.C., Samm-Vaughn, M.E., 2007. Hormonal correlates of human paternal interactions: a hospital based investigation in urban Jamaica. Horm. Behav. 52, 499-507.

Grewen, K.M., Girdler, S.S., Amico, J., Light, K.C., 2005. Effects of partner support on restring oxytocin, cortisol, norepinephrine and blood pressure before and after warm partner contact. Psychosom. Med. 67, 531-538.

Hammock, E.A.D., Young, L.J., 2002. Variation in vasopressin V1A receptor promotor and expression: implications for inter-and intra-specific variation in social behavior. Eur. J. Neurosci. 16, 399-402.

Hammock, E.A.D., Young, L.J., 2005. Microsatellite instability generates diversity in brain and sociobehavioral traits. Science 308, 1630-1634.
Insel, T.R., Shapiro, L.E., 1992. Oxytocin receptor distribution reflects social organization in monogamous and polygamous prairie voles. Proc. Nat. Acad. Sci. U.S.A. 89, 5981-5985.

Kosfeld, M., Heinrichs, M., Zak, P.J., Fischbacher, U., Fehr, E., 2005. Oxytocin increases trust in human. Nature 435, 673-676.

Kramer, K.M., Cushing, B.S., Carter, C.S., Wu, J., Ottinger, M.A., 2004. Sex and species differences in plasma oxytocin using an enzyme immunoassay. Can. J. Zool. 82 1194-1200.

Krüger, T.H.C., Haake, P., Chereath, D., Knapp, W., Janssen, O.E., Exton, M.S. Schedlowski, M., Hartmann, U., 2003. Specificity of the neuroendocrine response to orgasm during sexual arousal in men. J. Endocrinol. 177, 57-64.

Levine, A., Zagory-Sharon, O., Feldman, R., Weller, A., 2007. Oxytocin during pregnancy and early post-partum: individual patterns and maternal-fetal attachment. Peptides 28, 1162-1169.

Light, K.C., Grewen, K.M., Amico, J.A., 2005. More frequent partner hugs and higher oxytocin levels are liked to lower blood pressure and heart rate in premenopausal women. Biol. Psychol. 69, 5-21.

Lim, M.M., Young, L.J., 2006. Neuropeptidergic regulation of affiliative behavior and social bonding in animals. Horm. Behav. 50, 506-517.

Ochedalski, T., Subburaju, S., Wynn, P.C., Aguilera, G., 2007. Interaction between oestrogen and oxytocin on hypothalamic-pituitary-adrenal axis activity. J. Neuroendo. 19, 189-197.

Odendaal, J.S.J., Meintjes, R.A., 2003. Neurophysiological correlates of affiliative behavior between humans and dogs. Vet. J. 165, 296-301.

Pedersen, C.A., Prange Jr., A.J., 1979. Induction of maternal behavior in virgin rats after intracerebroventricular administration of oxytocin. Proc. Nat. Acad. Sci. U.S.A. 76, 6661-6665.

Ring, R.H., Malberg, J.E., Potestio, L., Ping, J., Boikess, S., Luo, B., Schechter, L.E., Rizzo, S., Rahman, Z., Rosenzweig-Lipson, S., 2006. Anxiolytic-like actions of oxytocin in male mice: behavioral and autonomic evidence: therapeutic implications. Psychopharm. $185,218-225$

Savage, A., Ziegler, T.E., Snowdon, C.T., 1988. Sociosexual development, pairbond formation and mechanisms of fertility suppression in female cotton-top tamarins (Saguinus oedipus oedipus). Am. J. Primatol. 14, 345-359.

Seltzer, L.J., Ziegler, T.E., 2007. Non-invasive measurement of small peptides in the common marmoset (Callithrix jacchus): a radiolabeled clearance study and endogenous excretion under varying social conditions. Horm. Behav. 51, 436-442.

Snowdon, C.T., Ziegler, T.E., 2007. Growing up cooperatively: family processes and infant development in marmosets and tamarins. J. Devel. Proc. 2, 40-66.

Strathearn, L., Fonagy, P., Amico, J., Montague, P.R., 2009. Adult attachment predicts maternal brain and oxytocin response to infant cues. Neuropsychopharm. 34, 2655-2666.

Taylor, S.E., Saphire-Bernstein, S., Seeman, T.E., 2010. Are plasma oxytocin in women and plasma vasopressin in men biomarkers of distressed pair relationships? Psychol. Sci. 21, 3-7.

Theodoridou, A., Rowe, A.C., Penton-Voak, I.S., Rogers, P.J., 2009. Oxytocin and social perception: oxytocin increases perceived facial trustworthiness and attractiveness. Horm. Behav. 56, 128-132.

Unkelbach, C., Guastella, A.J., Forgas, J.P., 2008. Oxytocin selectively facilitates recognition of positive sex and relationship words. Psych. Sci. 19, 1092-1094.

Uvnäs-Moberg, K., 1998. Oxytocin may mediate the benefits of positive social interaction and emotion. Psychoneuroendocrinology 23, 819-836.

Wallner, B., Dittami, J., Machatschke, I., 2006. Social stimuli cause changes in plasma oxytocin and behavior in guinea pigs. Biol. Res. 39, 251-258.

Walum, H., Westberg, L., Henningsson, S., Neiderhiser, J., Reiss, D., Igl, W., Ganiban, J.M., Spotts, E.L., Pedersen, N.L., Ericksson, E., Lichtenstein, P., 2008. Genetic variation in the vasopressin receptor 1a gene (AVPR1A) associates with pair-bonding behavior in humans. Proc. Nat. Acad. Sci. U.S.A. 105, 14153-14156.

Wang, Z., Zhou, L., Hulihan, T.J., Insel, T.R., 1996. Immunoreactivity of central vasopressin and oxytocin pathways in microtine rodents: a quantitative comparative study. J. Comp. Neurol. 366, 726-737.

Wang, Z., Moody, K., Newman, J.D., Insel, T.R., 1997. Vasopressin and oxytocin immunoreactive neurons and fibers in the forebrain of male and female common marmosets (Callithrix jacchus). Synapse 27, 14-25.

Wismer Fries, A.B., Ziegler, T.E., Kurian, J.R., Jacoris, S., Pollak, S.D., 2005. Early experience in humans is associated with changes in neuropeptides critical for regulating social behavior. Proc. Nat. Acad. Sci. U.S.A. 102, 17237-17240.

Zak, P.J., Kurzban, R., Matzner, W.T., 2005. Oxytocin is associated with human trustworthiness. Horm. Behav. 48, 522-527.

Ziegler, T.E., Savage, A., Scheffler, G., Snowdon, C.T., 1987. The endocrinology of puberty and reproductive functioning in female cotton-top tamarins (Saguinus oedipus) under varying social conditions. Biol. Reprod. 37, 618-627.

Ziegler, T.E., Scheffler, G., Snowdon, C.T., 1995. The relationship of cortisol levels to social environment and reproductive functioning in female cotton-top tamarins, Saguinus oedipus. Horm. Behav. 29, 407-424. 